\title{
Pulmonary Acantholytic Squamous Cell Carcinoma Mimicking Lepidic Pattern Adenocarcinoma
}

\section{Dear Editor,}

An 84-year-old male patient with a history of smoking and recent pneumonia symptoms of fever, difficulty in breathing, and weakness presented to the chest disease clinic. The patient had a left-sided suspicious lung mass on his chest X-Ray (CXR). Written informed consent was obtained from the patient. Chest computed tomography (CT) revealed a mass of $3 \times 2 \mathrm{~cm}$ on the superior lobe of the left lung (Figure 1). CT-guided transthoracic tru-cut biopsy of the chest was performed.

Microscopic examination revealed an infiltrative tumor consisting of atypical epithelial cells with a large hyperchromatic nucleus and showing prominent pleomorphism and acantholytic changes with gland-like structures in the lung parenchyma (Figure 2A,B). Immunohistochemical evaluation revealed diffuse positive staining with $\mathrm{p} 40$ and cytokeratin (CK) 7 (Figure 3A,B) while TTF-1, GATA-3 and CK20 were negative in the neoplastic cells. GATA-3 and CK20 immunohistochemical staining was performed as the tumor contained some urothelial carcinoma-like areas. Urothelial carcinoma metastasis was eliminated from the differential diagnosis as these markers were negative. A diagnosis of adenocarcinoma was eliminated and a diagnosis of squamous cell carcinoma was supported as a result of the negative staining with TTF-1 and diffuse strong positive staining with $\mathrm{p} 40$. With these findings, the case was reported as squamous cell carcinoma (acantholytic/ adenoid-like variant). An irregular bordered pathological lesion (SUVmax:10.4), with an axial long border of about $3 \mathrm{~cm}$, and creating traction on the mediastinal and costal pleura, was observed in the anterior segment of the superior lobe of the left lung. There was no evidence to suggest non-pulmonary SCC in other organs in the clinical or radiological signs. The patient was considered to be inoperable because of the distant metastases and chemotherapy treatment was planned.

SCC of the lung is a histological type of non-small cell lung carcinoma. It is one of the most prevalent lung cancers and originates from the bronchi. SCC infrequently presents with acantholysis, which is characterized by loosening of the cellto-cell connection. Acantholytic SCC is a rare variant, and arises most commonly in the skin. The acantholytic variant of SCC is rarely seen in the skin and also has an extremely rare presentation in the lung with only a few cases reported in the literature. Since the artefactual clefts of the tumoral cells could resemble acantholysis and glandular lumens or a vascular structure, acantholytic SCC is also called as adenoid / pseudovascular / pseudovascular adenoid SCC (1-3). We aimed to report our case as it is very rare in the literature.

Acantholytic SCC comprises 2-4\% of all cutaneous SCCs. Many skin pathology textbooks histologically characterize SCC as adenoid (pseudoglandular) or pseudoacinar nests with central acantholysis and cohesive peripheral tumor cells. The skin is the most frequent site of acantholytic tumors, with common skin pathology references. Pulmonary acantholytic SCC results in an aggressive clinical course, with marked lymphatic metastases $(4,5)$.p 40 has high immunohistochemical sensitivity and specificity to distinguish lung adenocarcinoma and SCC and appears to be an excellent marker for SCC. p40 immunostaining should be performed routinely for the diagnosis of pulmonary SCC (6). The positive expression rates of TTF1 and Napsin A are higher in lung adenocarcinoma, and TTF-1 is highly specific and sensitive in the diagnosis of adenocarcinoma. NapsinA may be used to distinguish $\mathrm{ADC}$ and SCC (7).

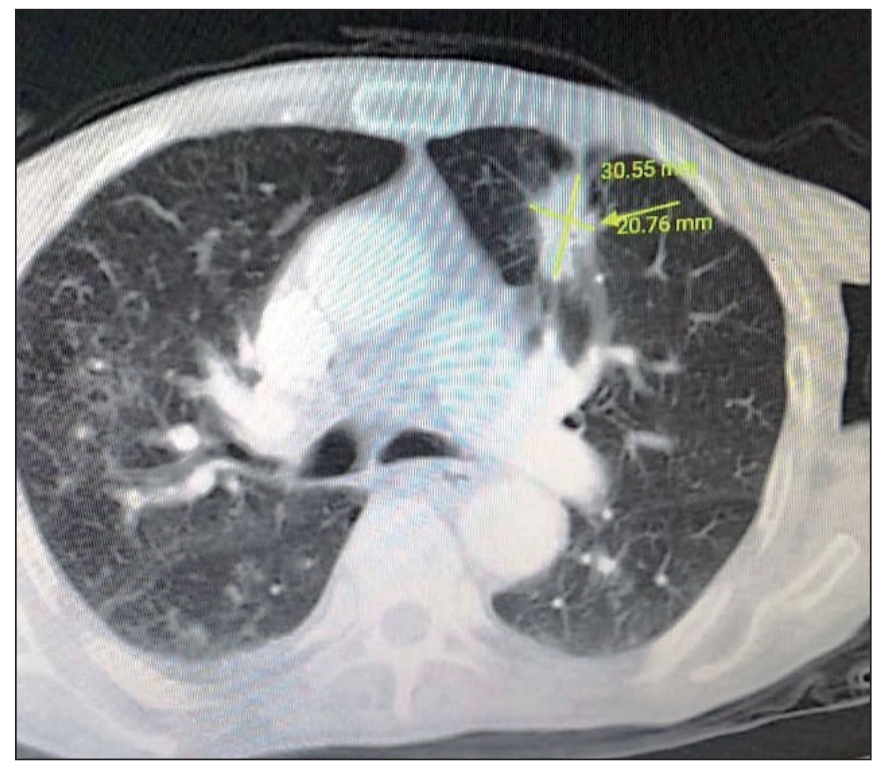

Figure 1: CT revealed a mass of $3 \times 2 \mathrm{~cm}$ on the superior lobe of the left lung (yellow arrow). 

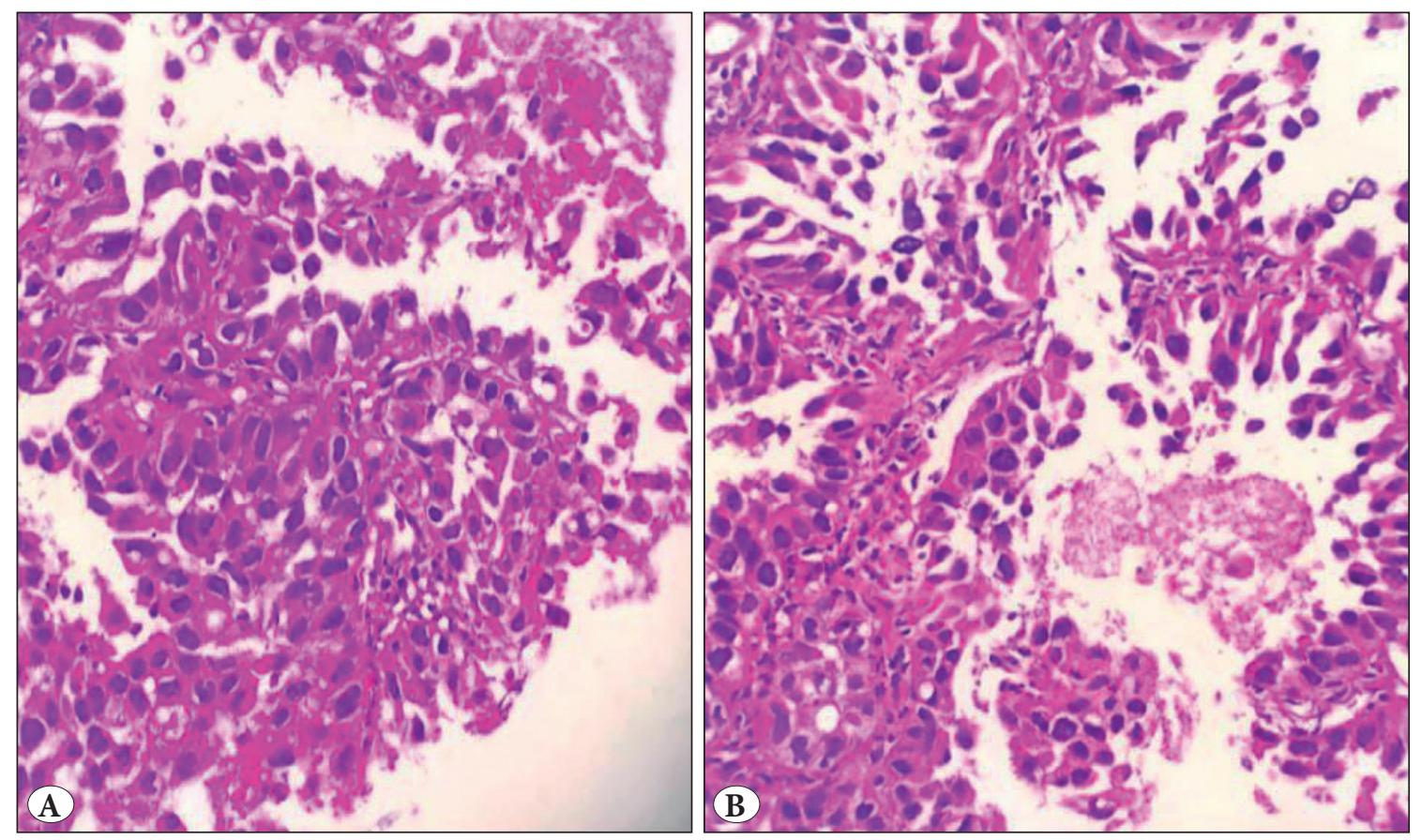

Figure 2: A-B) Atypical epithelial cells with large hyperchromatic and pleomorphic nucleus, showing acantholytic changes and forming gland-like structures (H\&E; x400).
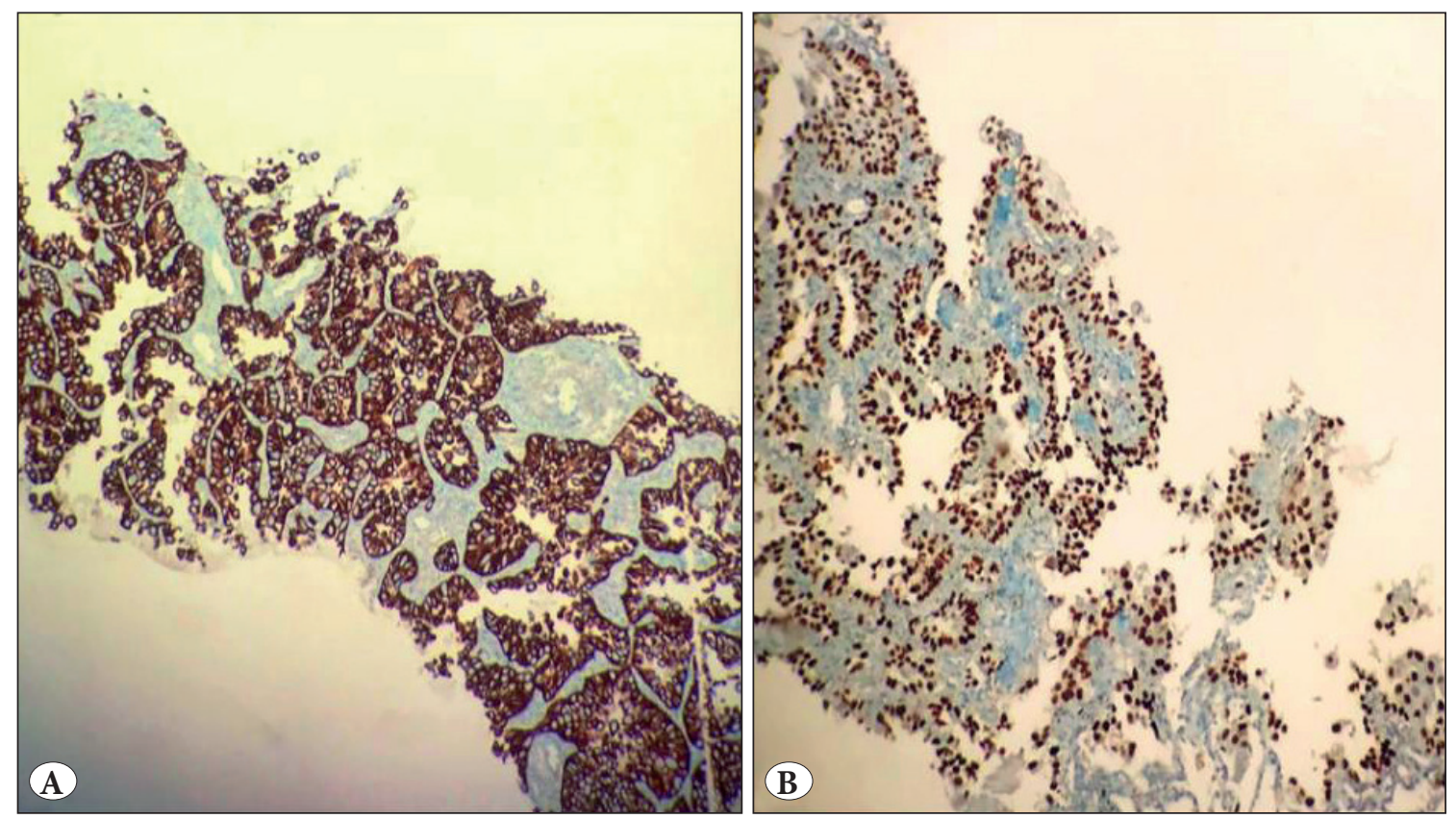

Figure 3: Neoplastic cells showed diffuse positivity for A) CK7 (IHC; x200) and B) p40 (IHC; x200)

In conclusion, the acantholytic/adenoid-like variant of SCC should be kept in mind during evaluation of lung biopsies, and careful microscopic examination and immunohistochemical evaluation should be performed since the tumor can mimic the lepidic pattern and other adenocarcinomas. The immunoexpression profile of SCC can help in making the correct diagnosis. 


\section{REFERENCES}

1. Weedon D, Morgan MB, Gross C, Nagore E, Yu LL. Squamous cell carcinoma. In: Le Boit PE, Burg G, Weedon D, Sarasin A, editors. World Health Organization classification of skin tumors. Lyon: International Agency for Research on Cancer; 2005. 20-5.

2. Choi SE, Park HS. Pulmonary acantholytic squamous cell carcinoma with focal signet ring cell morphology mimicking malignant mesothelioma on fine needle aspiration cytology: A case report. Cytopathology. 2016;27:131-3.

3. Park HS, Lee S. Acantholytic squamous cell carcinoma of the lung showing significant signet ring cell component. Histopathology. 2016;68:463-5.

4. Yiğit N, Çelik E, Yavan İ. Prominent signet ring cell morphology in a pulmonary squamous cell carcinoma. Turk Patoloji Derg. 2018;34:194-7.

5. Yorita K, Tsuji K, Takano Y, Kuroda N, Sakamoto K, Arii K, Yoshimoto Y, Nakatani K, Ito S. Acantholytic squamous cell carcinoma of the lung with marked lymphogenous metastases and high titers of myeloperoxidase-antineutrophil cytoplasmic antibodies: A case report. BMC Cancer. 2018;18:300.
6. Nobre AR, Albergaria A, Schmitt F. p40: A p63 isoform useful for lung cancer diagnosis - a review of the physiological and pathological role of p63. Acta Cytol. 2013;57:1-8.

7. Yu H, Li L, Liu D, Li WM. Expression of TTF-1, NapsinA, P63, $\mathrm{CK} 5 / 6$ in lung cancer and its diagnostic values for histological classification. Sichuan Da Xue Xue Bao Yi Xue Ban. 2017;48:33641.

\section{Rukiye YILMAZ (D)}

Recep Tayyip Erdoğan University School of Medicine,

Department of Pathology, RIZE, TURKEY

\section{Recep BEDİR}

Recep Tayyip Erdoğan University School of Medicine, Department of Pathology, RIZE, TURKEY

E-mail: bedirrecep@gmail.com

Phone: + 904642130491 